\title{
Hypophosphatemia after Treatment of Iron Deficiency with Intravenous Ferric Carboxymaltose or Iron Isomaltoside - A Systematic Review and Meta-Analysis
}

\author{
Benedikt Schaefer ${ }^{1,2}$, Moritz Tobiasch ${ }^{3}$, André Viveiros ${ }^{2}$, Herbert Tilg ${ }^{2}$, Nicholas A \\ Kennedy ${ }^{4}$, Myles Wolf ${ }^{5}$, and Heinz Zoller ${ }^{2}$ \\ ${ }^{1}$ Medizinische Universität Innsbruck \\ ${ }^{2}$ Medical University of Innsbruck \\ ${ }^{3}$ University Teaching Hospital of Hall in Tirol \\ ${ }^{4}$ University of Exeter \\ ${ }^{5}$ Duke University
}

September 11, 2020

\begin{abstract}
Aims: Hypophosphatemia is an increasingly recognized side-effect of ferric carboxymaltose (FCM) and possibly iron isomaltoside/ferric derisomaltose (IIM), which are used to treat iron deficiency. To determine frequency, severity, duration and risk factors of incident hypophosphatemia after treatment with FCM and IIM. Methods: A systematic literature search for articles indexed in EMBASE, PubMed and Web of Science in years 2005 to 2020 was carried out using the search terms 'ferric carboxymaltose' OR 'iron isomaltoside'. Prospective clinical trials reporting outcomes on hypophosphatemia rate, mean nadir serum phosphate and/or change in mean serum phosphate from baseline were selected. Hypophosphatemia rate and severity were compared for studies on IIM vs. FCM after stratification for chronic kidney disease. Meta-regression analysis was used to investigate risk factors for hypophosphatemia. Results: Across the 42 clinical trials included in the meta-analysis, FCM induced a significantly higher incidence of hypophosphatemia than IIM (47\%, 95\% CI 36-58\% vs. 4\%, 95\% CI $2-5 \%$ ), and significantly greater mean decreases in serum phosphate (0.40 versus $0.06 \mathrm{mmol} / \mathrm{L})$. Hypophosphatemia persisted at the end of the study periods (maximum 3 months) in up to $45 \%$ of patients treated with FCM. Meta-regression analysis identified low baseline serum ferritin and transferrin saturation, and normal kidney function as significant predictors of hypophosphatemia. Conclusion: FCM is associated with a high risk of hypophosphatemia, which does not resolve for at least 3 months in a large proportion of affected patients. More severe iron deficiency and normal kidney function are risk factors for hypophosphatemia.
\end{abstract}

Hypophosphatemia after Treatment of Iron Deficiency with Intravenous Ferric Carboxymaltose or Iron Isomaltoside - A Systematic Review and Meta-Analysis

Benedikt Schaefer, $\mathrm{MD}^{1,2}$, Moritz Tobiasch, $\mathrm{MD}^{3}$, André Viveiros, $\mathrm{MD}, \mathrm{PhD}^{1,2}$, Herbert Tilg, $\mathrm{MD}^{1}$, Nicholas A Kennedy, $\mathrm{PhD}^{4}$, Myles Wolf, MD, MMSc${ }^{5}$, Heinz Zoller, $\mathrm{MD}^{1,2}$

${ }^{1}$ Medical University of Innsbruck, Department of Medicine I, Gastroenterology, Hepatology and Endocrinology, Innsbruck, Austria

${ }^{2}$ Christian Doppler Laboratory of Iron and Phosphate Biology, Medical University of Innsbruck

${ }^{3}$ University Teaching Hospital of Hall in Tirol, Department of Medicine, Hall, Austria

${ }^{4}$ University of Exeter, IBD Pharmacogenetics, University of Exeter, Exeter, United Kingdom; Gastrointestinal Unit, Western General Hospital, Edinburgh, United Kingdom 
${ }^{5}$ Department of Medicine, Division of Nephrology, and Duke Clinical Research Institute, Duke University School of Medicine, Durham, NC, United States of America

Corresponding author: Heinz Zoller, MD

assoc. Professor of Medicine

Medical University of Innsbruck

Department of Medicine I, Gastroenterology, Hepatology and Endocrinology

Anichstrasse 35, A-6020 Innsbruck, Austria

Tel: +4351250481922 | Fax: +4351250428391

E-mail: heinz.zoller@i-med.ac.at, web: http://heplab.at

Short title: Hypophosphatemia after intravenous iron

Word count: 2336; 1761 (excluding abstract, methods, references and figure legends), Table count: 1 , Figure count: 4,Reference count: 63

Keywords: anaemia, anemia, phosphate, FGF23, ferric derisomaltose

\begin{abstract}
Aims: Hypophosphatemia is an increasingly recognized side-effect of ferric carboxymaltose (FCM) and possibly iron isomaltoside/ferric derisomaltose (IIM), which are used to treat iron deficiency. To determine frequency, severity, duration and risk factors of incident hypophosphatemia after treatment with FCM and IIM.

Methods: A systematic literature search for articles indexed in EMBASE, PubMed and Web of Science in years 2005 to 2020 was carried out using the search terms 'ferric carboxymaltose' OR 'iron isomaltoside'. Prospective clinical trials reporting outcomes on hypophosphatemia rate, mean nadir serum phosphate and/or change in mean serum phosphate from baseline were selected. Hypophosphatemia rate and severity were compared for studies on IIM vs. FCM after stratification for chronic kidney disease. Meta-regression analysis was used to investigate risk factors for hypophosphatemia.
\end{abstract}

Results : Across the 42 clinical trials included in the meta-analysis, FCM induced a significantly higher incidence of hypophosphatemia than IIM (47\%, 95\% CI 36-58\% vs. 4\%, 95\% CI 2-5\%), and significantly greater mean decreases in serum phosphate $(0.40$ versus $0.06 \mathrm{mmol} / \mathrm{L})$. Hypophosphatemia persisted at the end of the study periods (maximum 3 months) in up to $45 \%$ of patients treated with FCM. Meta-regression analysis identified low baseline serum ferritin and transferrin saturation, and normal kidney function as significant predictors of hypophosphatemia.

Conclusion : FCM is associated with a high risk of hypophosphatemia, which does not resolve for at least 3 months in a large proportion of affected patients. More severe iron deficiency and normal kidney function are risk factors for hypophosphatemia.

\title{
Introduction
}

Introduction of the intravenous iron preparations ferric carboxymaltose (FCM) and iron derisomaltose/iron isomaltoside 1000 (IIM) represented major advances in intravenous iron therapy because both preparations allow rapid correction of total iron deficit in one or two infusions, while exhibiting low rates of hypersensitivity reactions ${ }^{1,2}$. Both drugs are frequently used to treat iron deficiency of various causes. The commonest indications include iron deficiency due to gastrointestinal blood loss, inflammatory bowel disease (IBD), heavy uterine bleeding and post-partum hemorrhage ${ }^{3,4}$. Iron infusions are also frequently given to correct pre- or postoperative iron deficiency anemia. In patients with chronic kidney disease (CKD), i.v. iron increases 
response to erythropoiesis stimulating agents, while in patients with chronic heart failure, FCM improves exercise performance ${ }^{5-7}$.

Despite their overall favorable safety profile and simple administration schedule, hypophosphatemia is a sideeffect of both iron preparations ${ }^{8,9}$. Reporting of this side effect in clinical trials is inconsistent, leading to a remarkable variability in the documentation of hypophosphatemia rates. The severity of hypophosphatemia in affected patients is also underestimated when only mean phosphate concentrations across all patients is reported, including those who remain free from hypophosphatemia. The finding that hypophosphatemia persists in a subgroup of patients through the end of study follow-up, illustrates that the true duration of hypophosphatemia is unknown and can only be estimated from uncontrolled, retrospective studies ${ }^{10}$.

An increasing number of case reports suggests that hypophosphatemia after i.v. iron can cause acute, severe and/or chronic and potentially irreversible complications especially after repeated administration. Reported clinical manifestations include asthenia, fatigue, myopathy, respiratory failure, osteomalacia, bone pain and fractures, which are all recognized symptoms and signs of prolonged or severe hypophosphatemia ${ }^{11}$.

The mechanism of hypophosphatemia after i.v. iron is renal phosphate wasting; impaired kidney function partially protects against hypophosphatemia. Urinary phosphate excretion is regulated by the phosphaturic hormone, fibroblast-growth factor-23 (FGF23) ${ }^{12-14}$. Accordingly, the severity and risk of hypophosphatemia correlates with the magnitude of iron-induced increases in circulating concentrations of full-length, intact FGF23 (iFGF23) and with higher glomerular filtration rate ${ }^{11}$. Besides its effects as phosphaturic hormone, FGF23 also inhibits activation of $25(\mathrm{OH})$ vitamin D to $1,25(\mathrm{OH})_{2}$ vitamin D (calcitriol), which could explain the mild hypocalcemia and subsequent increase in circulating parathyroid hormone (PTH) concentration following intravenous iron treatment. Due to the phosphaturic effects of PTH, this mechanism may further prolong hypophosphatemia long after the increase in FGF23 returns towards normal ${ }^{15}$.

Differences in incidence, severity and possibly duration of hypophosphatemia, suggest that the specific pharmacological properties of the iron-carbohydrate complex may influence risk of hypophosphatemia ${ }^{11}$. Among i.v. iron preparations, saccharated iron oxide, iron sucrose and iron polymaltose, have been reported to induce hypophosphatemia ${ }^{16-19}$.

The diversity in the underlying etiology and variation in the severity of iron deficiency in patients included in different clinical trials limits direct comparison of safety data from prospective studies. To define the frequency and severity of hypophosphatemia after treatment with the internationally available high-dose intravenous iron preparations FCM and IIM, a systematic literature review and meta-analysis was carried out $^{20}$.

\section{Methods}

The rationale for this systematic review was to determine the incidence of hypophosphatemia after administration of FCM or IIM. To assess the incidence, we included prospective studies reporting on phosphate as a safety outcome. The search strategy is described in Figure 1 and the supplementary material. For the meta-analysis, data were extracted from each study by two authors independently. In case of inconsistent data entry, agreement was reached by discussion among authors. For each study included we recorded study drug and dose, the threshold for the definition of hypophosphatemia, the number of patients developing hypophosphatemia and the absolute number in whom phosphate was measured (safety analysis set) or, if available, hypophosphatemia rate and mean or median nadir phosphate. In addition, the following trial data were collected: the time point after administration of the study drug at which phosphate was measured, hypophosphatemia rate at the end of the study period and study duration. The following pre-treatment parameters were assessed in the FCM or IIM treatment arms: median or mean concentration of ferritin, hemoglobin, transferrin saturation and phosphate concentration. A single study reported on relevant outcomes from a direct head to head comparison of IIM and FCM. For this meta-analysis of safety outcomes data on hypophosphatemia for the FCM or IIM treatment arm were assessed for each study individually. Due to the wide range of treatments in the comparator arms (placebo, standard medical care, various oral iron 
preparations, ferrumoxytol, iron dextran, iron sucrose, saccharated iron oxide) odds ratios are not comparable between studies. Criteria for risk bias assessment were predefined to consider potential limitations in study design and reporting that could potentially affect observed hypophosphatemia rate or severity. Each of studies included was assessed according to the criteria listed in Figure S1. According to these criteria each study was graded and the overall score correlated with outcomes reported by Spearman rank correlation analysis. Risk of publication bias was assessed by funnel plot analysis.

The meta-analysis was performed using the metafor package (version 2.1-0) in $\mathrm{R}(\mathrm{R}$ version, 3.6.1 (R Foundation for Statistical Computing, Vienna, Austria) and RStudio (version 1.2.5001, RStudio, Inc., Boston, MA, USA) as described by Viechtbauer et $\mathrm{al}^{21}$. Effect sizes of frequencies and counts were calculated as raw proportions. In case of zeroes, a value of 0.01 was added to the respective value to avoid division by zero; all other values remained unmodified. Effect sizes of mean phosphate levels and mean change of phosphate were calculated as mean differences of reported mean post-treatment phosphate minus mean pre-treatment phosphate concentration. For subgrouping, studies were stratified by iron preparation and the presence or absence of chronic kidney disease in the study population. Heterogeneity between studies was estimated by a restricted maximum-likelihood estimator and reported using the Cochrane $\mathrm{Q}$ and the $\mathrm{I}^{2}$ statistic. Additional details of the statistical methods can be found in the supplementary material.

For meta-regressions, mean serum ferritin, mean transferrin saturation, and mean age were extracted from the studies' baseline characteristics. For ferritin, a log-linear weighted regression was performed, whereas transferrin saturation was treated as a potential linear modifier. The respective $\mathrm{R}$ syntax is given in the appendix. Serum ferritin values were $\log 10$ transformed for further computations, as this parameter follows a log-normal distribution.

Frequencies were either calculated by dividing the number of patients developing hypophosphatemia by the number of patients in whom phosphate concentrations were available or the hypophosphatemia rate as reported in the study.

\section{Results}

\section{Hypophosphatemia rates in prospective studies}

The systematic literature search identified 224 prospective studies. After manual selection, 42 clinical trials that used either FCM or IIM and reported relevant outcomes on phosphate were included in the meta-analysis (Figure 1). Outcomes were inconsistently reported (Table 1). Seventeen of 42 studies defined hypophosphatemia as a serum phosphate concentration $<0.64 \mathrm{mmol} / \mathrm{L}$ or $<2.0 \mathrm{mg} / \mathrm{dL}$ ) and 8 of 42 studies used serum phosphate up to $0.84 \mathrm{mmol} / \mathrm{L}(2.56 \mathrm{mg} / \mathrm{dl})$ as cutoff. To assess if the frequency of hypophosphatemia depended on the threshold applied, this variable was included in the risk-bias assessment (Figure S1). Twenty-five of 42 studies included in the final analysis reported a threshold for the definition of hypophosphatemia. Other criteria that were included in the risk-bias assessment were the point in time when the phosphate was measured, mean nadir phosphate and hypophosphatemia rate at the end of follow-up. Only four studies reported all relevant definitions and outcomes on hypophosphatemia (Figure S1). Risk bias assessment showed that studies with higher risk score reported significantly lower hypophosphatemia rates $(\mathrm{r}=0.348, \mathrm{p}=0.021)$. No significant correlation between overall risk score, mean change in phosphate or mean nadir phosphate was found. Independent analysis of studies on FCM and IIM by funnel blot analysis showed no evidence of publication bias (Figure S2).

Hypophosphatemia rates ranged from $0 \%$ to $92 \%$. To assess to what extend heterogeneity could be explained by use of IIM or FCM, studies were grouped by study population (CKD vs. non-CKD). The pooled hypophosphatemia rate after FCM was significantly higher than after IIM (47\% vs. 4\%; p <0.001). Since reduced glomerular filtration rate impairs urinary phosphate excretion, we next assessed kidney function as a cause of heterogeneity, which was reduced by analyzing CKD patients independently. In CKD patients, hypophosphatemia rates were $27 \%$ after FCM vs. $2 \%$ after IIM (Figure 2). Despite significant heterogeneity 
among studies in non-CKD-patients, pooled hypophosphatemia rates were significantly higher after FCM than after IIM treatment ( 51 vs $5 \%$; $\mathrm{p}<0.001$ ).

Studies reporting mean nadir plasma phosphate concentrations in the treatment arms after administration of FCM or IIM are shown in Figure 3A. Pooled analysis shows that in few studies mean nadir phosphate reaches a concentration $<0.6 \mathrm{mmol} / \mathrm{L}$. The overall pooled mean nadir phosphate concentration after FCM was $0.69 \mathrm{mmol} / \mathrm{L}(95 \%$ CI $0.60-0.78)$ in patients without CKD vs. $1.11 \mathrm{mmol} / \mathrm{L}$ (95\% CI $0.96-1.27$ ) in the subgroup of patients with CKD.

Figure 3B shows analysis of mean change in phosphate from baseline. The pooled analysis of reported results across etiologies shows that the mean decrease in phosphate after FCM is -0.40 (95\% CI -0.50- -0.31$) \mathrm{mmol} / \mathrm{L}$ vs. 0.06 (95\% CI -0.14-0.02) mmol/L after IIM.

The duration of hypophosphatemia was not reported in any of the studies, but hypophosphatemia rate at the end of the study period ranged from $0-45 \%$ after a follow-up of up to 3 months (Table 1 ).

Meta-regression analysis was carried out to test if serum iron parameters are predictors of hypophosphatemia. As shown in Figure 4, serum ferritin and transferrin saturation at baseline showed a significant association with a hypophosphatemia rate after stratification of trials by study drug. In trials on both study drugs, more severe iron deficiency was associated with a greater risk of post-treatment hypophosphatemia, but the overall risk for hypophosphatemia was lower for IIM. A stronger negative association between hypophosphatemia rate and $\log$ (ferritin) was noted for FCM than for IIM. The same was observed for the association between transferrin saturation and hypophosphatemia rate (Figure 4, Table S1). No association between mean total iron dose and hypophosphatemia rate was found when studies using FCM or IIM were analyzed independently. When mean total iron dose was correlated with nadir phosphate concentration, a significant association was found on meta-regression analysis (Figure S3).

\section{Discussion}

Despite the advantages of latest generation intravenous preparations FCM and IIM, which allow safe correction of total iron deficit in 1-2 infusions in the majority of patients, hypophosphatemia is increasingly recognized as a delayed adverse drug reaction. Although listed in the drug label as a potential side effect, the overall incidence and the clinical relevance of this adverse event are largely unknown. ${ }^{11,22,23}$

This systematic review and meta-analysis show that the reported incidence of hypophosphatemia after infusion of FCM or IIM ranges from $0 \%$ to $92 \%$ of in prospective clinical trials. Our systematic review and meta-analysis show that this wide range is in part attributable to inconsistent assessment and reporting of hypophosphatemia in clinical studies. As determinants and predictors of hypophosphatemia we have identified preserved renal function, the severity of iron deficiency and possibly dose.

The present analysis highlights inconsistencies in reporting of relevant endpoints. Some studies only report on mean phosphate concentrations before and after treatment or relative changes. Incidence of hypophosphatemia and the proportion of patients developing severe hypophosphatemia $(<0.3 \mathrm{mmol} / \mathrm{L})$ are much more relevant for the care of individual patients. Study protocols of ongoing clinical trials, which include assessment of the prevalence of the different degrees of hypophosphatemia $(<0.3 \mathrm{mmol} / \mathrm{L}, 0.3-0.6 \mathrm{mmol} / \mathrm{L}$, 0.6-0.8 $\mathrm{mmol} / \mathrm{L}$ ) during follow up visits are preferable and should become standard in reporting this side effect, because the risk for complications of hypophosphatemia increases with its severity ${ }^{24}$.

Further, the duration of hypophosphatemia should be prospectively assessed by following-up on patients who develop hypophosphatemia during the study. Accordingly, the description of 'i.v. iron-induced hypophosphatemia' as 'transient decrease of phosphate levels' may be applicable to a study population but inappropriate in describing the side-effect in individual patients, because recovery is unpredictable for individual patients. Retrospective studies have suggested that the median time to recovery is $40-80$ days, but this is insufficient to assess the true duration of hypophosphatemia in individual patients, who developed this side-effect ${ }^{10}$. 
None of the prospective studies reported on the actual duration of hypophosphatemia. Hence, the risk of long-term complications is also unknown.

Among patients with impaired renal function, the pooled risk of hypophosphatemia was significantly lower when compared with studies in other patient populations. The result supports previous findings that FCM and to a lesser degree IIM cause hypophosphatemia by renal wasting. Urinary phosphate excretion is controlled by the hormone intact FGF23, which is induced by FCM through incompletely defined mechanisms ${ }^{25}$.

The difference in incidence between FCM and IIM across etiologies supports the notion that hypophosphatemia is not a class effect of all i.v. irons, but rather a specific effect of FCM. This conclusion is supported by clinical trials confirming that despite comparable dosing of FCM and the comparator (iron dextran or ferumoxytol), hypophosphatemia was far more common after FCM ${ }^{15,26}$. Pharmacologically, i.v. iron preparations are 'non-biological complex drugs (NBCD)', where the active drug is not a homo-molecular structure but consists of different closely related structures. The composition, characteristics and in-vivo effects of NBCD are highly dependent on manufacturing processes, which limits the possibilities to study the carbohydrate moiety independently of iron ${ }^{27}$.

The clinical manifestations of inappropriately high FGF23 with consequent hypophosphatemia are diverse and best known from patients with defects in genes regulating phosphate homeostasis and patients with tumor-induced osteomalacia. Weakness of proximal muscles, dental problems, bone pain and osteomalacia are typical manifestations of these diseases. As treatment with certain i.v. iron preparations is a novel cause of inappropriate FGF23 elevation, it can be expected that severely and chronically affected patients could also develop symptoms mimicking TIO or genetic hypophosphatemia ${ }^{28}$. Accordingly, a number of cases with clinical complications of hypophosphatemia has been reported ${ }^{11}$.

\section{Conclusion}

In conclusion, this systematic review and meta-analysis shows that hypophosphatemia is a common medical problem after administration of FCM, especially in patients with preserved kidney function and more severe iron deficiency. The incidence is higher, severity and duration are more severe after FCM as compared to IIM.

Abbrevations: FCM, ferric carboxymaltose; IIM, iron isomaltoside; FS, ferric sucrose; SIO, saccharated iron oxide; ID, iron dextrane; FO, ferumoxytol, na, not available; GYN, gynecology; IDA, iron defiecency anemia; PBM, patient blood management; CKD, chronic kidney disease; NDD-CKD, non-dialysis dependent chronic kidney disease; HD-CKD, hemodialysis-dependent chronic kidney disease; RLS, restless leg syndrome

Acknowledgment/Conflict of interest: Dr Schaefer reported receiving personal fees from Pharmacosmos A/S, Vifor Pharma, grants and personal fees from Abbvie and Gilead outside the submitted work.

Dr Wolf reported receiving personal fees from Pharmacosmos A/S, AMAG Pharmaceuticals, Amgen, Akebia, Ardelyx, Keryx, and Luitpold Inc. outside the submitted work.

Dr Zoller reported receiving grants, personal fees, and nonfinancial support from Pharmacosmos A/S, Vifor Pharma, Abbvie and Gilead; personal fees from Merck; personal fees and nonfinancial support from Bayer; grants from Merck Sharp \& Dohme; and honoraria for lecturing from Bristol-Myers Squibb, Merz, Medice, Novartis outside the submitted work.

Funding: Christian Doppler Laboratory for Iron and Phosphate Biology, Medical University of Innsbruck, Department of Medicine I, Anichstr. 35, A-6020 Innsbruck, Austria.

For original data, please contact the corresponding author.

\section{References}

1. Onken JE, Bregman DB, Harrington RA, et al. A multicenter, randomized, active-controlled study to investigate the efficacy and safety of intravenous ferric carboxymaltose in patients with iron deficiency anemia. 
Transfusion . 2014;54(2):306-315.

2. Stein J, Walper A, Klemm W, Farrag K, Aksan A, Dignass A. Safety and efficacy of intravenous iron isomaltoside for correction of anaemia in patients with inflammatory bowel disease in everyday clinical practice.Scand J Gastroenterol . 2018;53(9):1059-1065.

3. Kalra PA, Bhandari S. Efficacy and safety of iron isomaltoside (Monofer $((\mathrm{R}))$ ) in the management of patients with iron deficiency anemia. Int J Nephrol Renovasc Dis . 2016;9:53-64.

4. Moore RA, Gaskell H, Rose P, Allan J. Meta-analysis of efficacy and safety of intravenous ferric carboxymaltose (Ferinject) from clinical trial reports and published trial data. BMC Blood Disord . 2011;11:4.

5. Cunha GJL, Rocha BML, Menezes Falcao L. Iron deficiency in chronic and acute heart failure: A contemporary review on intertwined conditions. Eur J Intern Med . 2018;52:1-7.

6. Keating GM. Ferric carboxymaltose: a review of its use in iron deficiency. Drugs . 2015;75(1):101-127.

7. Qunibi WY. The efficacy and safety of current intravenous iron preparations for the management of iron-deficiency anaemia: a review.Arzneimittelforschung . 2010;60(6a):399-412.

8. Van Wyck DB, Martens MG, Seid MH, Baker JB, Mangione A. Intravenous ferric carboxymaltose compared with oral iron in the treatment of postpartum anemia: a randomized controlled trial. Obstet Gynecol . 2007;110(2 Pt 1):267-278.

9. Reinisch W, Staun M, Tandon RK, et al. A randomized, open-label, non-inferiority study of intravenous iron isomaltoside 1,000 (Monofer) compared with oral iron for treatment of anemia in IBD (PROCEED). Am J Gastroenterol . 2013;108(12):1877-1888.

10. Schaefer B, Wurtinger P, Finkenstedt A, et al. Choice of High-Dose Intravenous Iron Preparation Determines Hypophosphatemia Risk.PLoS One . 2016;11(12):e0167146.

11. Zoller H, Schaefer B, Glodny B. Iron-induced hypophosphatemia: an emerging complication. Curr Opin Nephrol Hypertens . 2017;26(4):266-275.

12. Gravesen E, Hofman-Bang J, Mace ML, Lewin E, Olgaard K. High dose intravenous iron, mineral homeostasis and intact FGF23 in normal and uremic rats. BMC Nephrol . 2013;14:281.

13. Prats M, Font R, Garcia C, Cabre C, Jariod M, Vea AM. Effect of ferric carboxymaltose on serum phosphate and C-terminal FGF23 levels in non-dialysis chronic kidney disease patients: post-hoc analysis of a prospective study. BMC Nephrol . 2013;14:167.

14. Roberts MA, Huang L, Lee D, et al. Effects of intravenous iron on fibroblast growth factor 23 (FGF23) in haemodialysis patients: a randomized controlled trial. BMC Nephrol . 2016;17(1):177.

15. Wolf M, Koch TA, Bregman DB. Effects of iron deficiency anemia and its treatment on fibroblast growth factor 23 and phosphate homeostasis in women. J Bone Miner Res . 2013;28(8):1793-1803.

16. Sato K, Shiraki M. Saccharated ferric oxide-induced osteomalacia in Japan: iron-induced osteopathy due to nephropathy. Endocr J . 1998;45(4):431-439.

17. Schouten BJ, Doogue MP, Soule SG, Hunt PJ. Iron polymaltose-induced FGF23 elevation complicated by hypophosphataemic osteomalacia. Ann Clin Biochem . 2009;46(Pt 2):167-169.

18. Schouten BJ, Hunt PJ, Livesey JH, Frampton CM, Soule SG. FGF23 elevation and hypophosphatemia after intravenous iron polymaltose: a prospective study. J Clin Endocrinol Metab . 2009;94(7):2332-2337.

19. Hardy S, Vandemergel X. Intravenous iron administration and hypophosphatemia in clinical practice. Int $J$ Rheumatol . 2015;2015:468675.

20. Schaefer B, Glodny B, Wolf M, Zoller H. Letter: inconsistency in reporting of hypophosphatemia after intravenous iron. Aliment Pharmacol Ther . 2017;46(6):641-643. 
21. Viechtbauer W. Conducting meta-analyses in $\mathrm{R}$ with the metafor package. Journal of statistical software . 2010;36(3):1-48.

22. Mace TA, Syed A, Bhandari S. Iron (III) isomaltoside 1000. Expert Rev Hematol . 2013;6(3):239-246.

23. Cada DJ, Levien TL, Baker DE. Ferric carboxymaltose. Hosp Pharm . 2014;49(1):52-59.

24. Geerse DA, Bindels AJ, Kuiper MA, Roos AN, Spronk PE, Schultz MJ. Treatment of hypophosphatemia in the intensive care unit: a review. Critical Care . 2010;14(4):R147.

25. Vervloet M. Renal and extrarenal effects of fibroblast growth factor 23. Nat Rev Nephrol . 2019;15(2):109120.

26. Wolf M, Chertow GM, Macdougall IC, Kaper R, Krop J, Strauss W. Randomized trial of intravenous iron-induced hypophosphatemia. JCI Insight . 2018;3(23).

27. Schellekens H, Stegemann S, Weinstein V, et al. How to regulate nonbiological complex drugs (NBCD) and their follow-on versions: points to consider. AAPS J . 2014;16(1):15-21.

28. Edmonston D, Wolf M. FGF23 at the crossroads of phosphate, iron economy and erythropoiesis. Nat Rev Nephrol . 2020;16(1):7-19.

29. Allen RP, Adler CH, Du W, Butcher A, Bregman DB, Earley CJ. Clinical efficacy and safety of IV ferric carboxymaltose (FCM) treatment of RLS: a multi-centred, placebo-controlled preliminary clinical trial.Sleep Med . 2011;12(9):906-913.

30. Auerbach M, Henry D, Derman RJ, Achebe MM, Thomsen LL, Glaspy J. A prospective, multi-center, randomized comparison of iron isomaltoside 1000 versus iron sucrose in patients with iron deficiency anemia; the FERWON-IDA trial. Am J Hematol . 2019.

31. Bailie GR, Mason NA, Valaoras TG. Safety and tolerability of intravenous ferric carboxymaltose in patients with iron deficiency anemia. Hemodial Int . 2010;14(1):47-54.

32. Barish CF, Koch T, Butcher A, Morris D, Bregman DB. Safety and Efficacy of Intravenous Ferric Carboxymaltose $(750 \mathrm{mg}$ ) in the Treatment of Iron Deficiency Anemia: Two Randomized, Controlled Trials.Anemia . 2012;2012:172104.

33. Bhandari S, Kalra PA, Kothari J, et al. A randomized, open-label trial of iron isomaltoside 1000 (Monofer(R)) compared with iron sucrose (Venofer(R)) as maintenance therapy in haemodialysis patients. Nephrol Dial Transplant . 2015;30(9):1577-1589.

34. Bhandari S, Kalra PA, Berkowitz M, Belo D, Thomsen LL, Wolf M. Safety and efficacy of iron isomaltoside 1000/ferric derisomaltose versus iron sucrose in patients with chronic kidney disease: the FERWONNEPHRO randomized, open-label, comparative trial. Nephrol Dial Transplant . 2020.

35. Biggar P, Leistikow F, Walper A. A prospective observational study of effectiveness and safety of iron isomaltoside in patients with chronic renal failure and iron deficiency anemia. Clin Nephrol . 2016;86 (2016)(12):310-318.

36. Birgegard G, Henry D, Glaspy J, Chopra R, Thomsen LL, Auerbach M. A Randomized Noninferiority Trial of Intravenous Iron Isomaltoside versus Oral Iron Sulfate in Patients with Nonmyeloid Malignancies and Anemia Receiving Chemotherapy: The PROFOUND Trial. Pharmacotherapy . 2016;36(4):402-414.

37. Breymann C, Milman N, Mezzacasa A, Bernard R, Dudenhausen J, investigators F-A. Ferric carboxymaltose vs. oral iron in the treatment of pregnant women with iron deficiency anemia: an international, open-label, randomized controlled trial (FER-ASAP). J Perinat Med . 2017;45(4):443-453.

38. Charytan C, Bernardo MV, Koch TA, Butcher A, Morris D, Bregman DB. Intravenous ferric carboxymaltose versus standard medical care in the treatment of iron deficiency anemia in patients with chronic kidney disease: a randomized, active-controlled, multi-center study.Nephrol Dial Transplant . 2013;28(4):953-964. 
39. Dahlerup JF, Jacobsen BA, van der Woude J, Bark LA, Thomsen LL, Lindgren S. High-dose fast infusion of parenteral iron isomaltoside is efficacious in inflammatory bowel disease patients with iron-deficiency anaemia without profound changes in phosphate or fibroblast growth factor 23. Scand J Gastroenterol . 2016;51(11):1332-1338.

40. Detlie TE, Lindstrom JC, Jahnsen ME, et al. Incidence of hypophosphatemia in patients with inflammatory bowel disease treated with ferric carboxymaltose or iron isomaltoside. Aliment Pharmacol Ther . 2019;50(4):397-406.

41. Ding Y, Zhu X, Li X, et al. Pharmacokinetic, Pharmacodynamic, and Safety Profiles of Ferric Carboxymaltose in Chinese Patients with Iron-Deficiency Anemia. Clin Ther . 2020.

42. Drexler C, Macher S, Lindenau I, et al. High-dose intravenous versus oral iron in blood donors with iron deficiency: The IronWoMan randomized, controlled clinical trial. Clin Nutr . 2019.

43. Evstatiev R, Marteau P, Iqbal T, et al. FERGIcor, a randomized controlled trial on ferric carboxymaltose for iron deficiency anemia in inflammatory bowel disease. Gastroenterology . 2011;141(3):846-853 e841-842.

44. Favrat B, Balck K, Breymann C, et al. Evaluation of a single dose of ferric carboxymaltose in fatigued, iron-deficient women-PREFER a randomized, placebo-controlled study. PLoS One . 2014;9(4):e94217.

45. Gybel-Brask M, Seeberg J, Thomsen LL, Johansson PI. Intravenous iron isomaltoside improves hemoglobin concentration and iron stores in female iron-deficient blood donors: a randomized double-blind placebo-controlled clinical trial. Transfusion . 2018;58(4):974-981.

46. Holm C, Thomsen LL, Norgaard A, Langhoff-Roos J. Single-dose intravenous iron infusion or oral iron for treatment of fatigue after postpartum haemorrhage: a randomized controlled trial. Vox Sang . 2017;112(3):219-228.

47. Huang LL, Lee D, Troster SM, et al. A controlled study of the effects of ferric carboxymaltose on bone and haematinic biomarkers in chronic kidney disease and pregnancy. Nephrol Dial Transplant . 2018;33(9):16281635 .

48. Hussain I, Bhoyroo J, Butcher A, Koch TA, He A, Bregman DB. Direct Comparison of the Safety and Efficacy of Ferric Carboxymaltose versus Iron Dextran in Patients with Iron Deficiency Anemia. Anemia . 2013;2013:169107.

49. Ikuta K, Shimura A, Terauchi M, Yoshii K, Kawabata Y. Pharmacokinetics, pharmacodynamics, safety, and tolerability of intravenous ferric carboxymaltose: a dose-escalation study in Japanese volunteers with iron-deficiency anemia. Int J Hematol . 2018;107(5):519-527.

50. Ikuta K, Hanashi H, Hirai K, et al. Comparison of efficacy and safety between intravenous ferric carboxymaltose and saccharated ferric oxide in Japanese patients with iron-deficiency anemia due to hypermenorrhea: a multi-center, randomized, open-label noninferiority study. Int J Hematol . 2019;109(1):41-49.

51. Ikuta K, Ito H, Takahashi K, Masaki S, Terauchi M, Suzuki Y. Safety and efficacy of intravenous ferric carboxymaltose in Japanese patients with iron-deficiency anemia caused by digestive diseases: an open-label, single-arm study. Int J Hematol . 2019;109(1):50-58.

52. Johansson PI, Rasmussen AS, Thomsen LL. Intravenous iron isomaltoside 1000 (Monofer(R)) reduces postoperative anaemia in preoperatively non-anaemic patients undergoing elective or subacute coronary artery bypass graft, valve replacement or a combination thereof: a randomized double-blind placebo-controlled clinical trial (the PROTECT trial). Vox Sang . 2015;109(3):257-266.

53. Kalra PA, Bhandari S, Saxena S, et al. A randomized trial of iron isomaltoside 1000 versus oral iron in nondialysis-dependent chronic kidney disease patients with anaemia. Nephrol Dial Transplant . 2016;31(4):646655. 
54. Macdougall IC, Bock A, Carrera F, et al. The FIND-CKD study-a randomized controlled trial of intravenous iron versus oral iron in non-dialysis chronic kidney disease patients: background and rationale.Nephrol Dial Transplant . 2014;29(4):843-850.

55. Mahey R, Kriplani A, Mogili KD, Bhatla N, Kachhawa G, Saxena R. Randomized controlled trial comparing ferric carboxymaltose and iron sucrose for treatment of iron deficiency anemia due to abnormal uterine bleeding. Int J Gynaecol Obstet . 2016;133(1):43-48.

56. Onken JE, Bregman DB, Harrington RA, et al. Ferric carboxymaltose in patients with iron-deficiency anemia and impaired renal function: the REPAIR-IDA trial. Nephrol Dial Transplant . 2014;29(4):833-842.

57. Qunibi WY, Martinez C, Smith M, Benjamin J, Mangione A, Roger SD. A randomized controlled trial comparing intravenous ferric carboxymaltose with oral iron for treatment of iron deficiency anaemia of nondialysis-dependent chronic kidney disease patients. Nephrol Dial Transplant . 2011;26(5):1599-1607.

58. Seid MH, Derman RJ, Baker JB, Banach W, Goldberg C, Rogers R. Ferric carboxymaltose injection in the treatment of postpartum iron deficiency anemia: a randomized controlled clinical trial. Am J Obstet Gynecol . 2008;199(4):435 e431-437.

59. Seid MH, Butcher AD, Chatwani A. Ferric Carboxymaltose as Treatment in Women with Iron-Deficiency Anemia. Anemia . 2017;2017:9642027.

60. Stohr R, Sandstede L, Heine GH, Marx N, Brandenburg V. High-Dose Ferric Carboxymaltose in Patients With HFrEF Induces Significant Hypophosphatemia. J Am Coll Cardiol . 2018;71(19):2270-2271.

61. Toledano A, Luporsi E, Morere JF, et al. Clinical use of ferric carboxymaltose in patients with solid tumours or haematological malignancies in France. Support Care Cancer . 2016;24(1):67-75.

62. Van Wyck DB, Mangione A, Morrison J, Hadley PE, Jehle JA, Goodnough LT. Large-dose intravenous ferric carboxymaltose injection for iron deficiency anemia in heavy uterine bleeding: a randomized, controlled trial. Transfusion . 2009;49(12):2719-2728.

63. Wolf M, Rubin J, Achebe M, et al. Effects of Iron Isomaltoside vs Ferric Carboxymaltose on Hypophosphatemia in Iron-Deficiency Anemia: Two Randomized Clinical Trials. JAMA . 2020;323(5):432-443.

\section{Tables}

Table 1: Characteristics of the 42 prospective clinical trials Included in the meta-analysis.

\begin{tabular}{|c|c|c|c|}
\hline First author (year) & Study type & Therapeutic area & Threshold for hypophosphatemia \\
\hline Allen et al. $(2011)^{29}$ & $\mathrm{RCT}$ & RLS & na \\
\hline Auerbach et al. $(2019)^{30}$ & $\mathrm{RCT}$ & mixed IDA & $2 \mathrm{mg} / \mathrm{dL}(0.65 \mathrm{mmol} / \mathrm{L}$ \\
\hline Bailie et al. $(2010)^{31}$ & $\mathrm{RCT}$ & mixed IDA & na \\
\hline Barish et al. $(2012)^{32}-$ multiple dose arm & $\mathrm{RCT}$ & mixed IDA & $2 \mathrm{mg} / \mathrm{dL}(0.65 \mathrm{mmol} / \mathrm{L})$ \\
\hline Barish et al. $(2012)^{32}-$ single dose arm & $\mathrm{RCT}$ & mixed IDA & $2 \mathrm{mg} / \mathrm{dL}(0.65 \mathrm{mmol} / \mathrm{L})$ \\
\hline Bhandari et al. $(2015)^{33}$ & $\mathrm{RCT}$ & HD-CKD & $2 \mathrm{mg} / \mathrm{dL}(0.65 \mathrm{mmol} / \mathrm{L})$ \\
\hline Bhandari et al. $(2020)^{34}$ & $\mathrm{RCT}$ & NDD-CKD & $2 \mathrm{mg} / \mathrm{dL}(0.65 \mathrm{mmol} / \mathrm{L})$ \\
\hline Biggar et al. $(2016)^{35}$ & SAT & HD-CKD & na \\
\hline Birgegard et al. $(2016)^{36}$ - bolus arm & $\mathrm{RCT}$ & malignancy & $2 \mathrm{mg} / \mathrm{dL}(0.65 \mathrm{mmol} / \mathrm{L})$ \\
\hline Birgegard et al. $(2016)^{36}$ - infusion arm & $\mathrm{RCT}$ & malignancy & $2 \mathrm{mg} / \mathrm{dL}(0.65 \mathrm{mmol} / \mathrm{L})$ \\
\hline Breymann et al. $(2017)^{37}$ & $\mathrm{RCT}$ & GYN & $0.6 \mathrm{mmol} / \mathrm{L}$ \\
\hline Charytan et al. $(2013)^{38}-$ HD-arm & $\mathrm{RCT}$ & HD-CKD & na \\
\hline Charytan et al. $(2013)^{38}-$ NDD-HD arm & $\mathrm{RCT}$ & NDD-CKD & na \\
\hline Dahlerup et al. $(2016)^{39}$ & SAT & IDA digestive diseases & $2 \mathrm{mg} / \mathrm{dL}(0.65 \mathrm{mmol} / \mathrm{L})$ \\
\hline
\end{tabular}




\begin{tabular}{|c|c|c|c|}
\hline First author (year) & Study type & Therapeutic area & Threshold for hypophosphatemia \\
\hline Detlie et al. $(2019)^{40}$ - FCM arm & $\mathrm{RCT}$ & IDA digestive diseases & $0.8 \mathrm{mmol} / \mathrm{L}$ \\
\hline Detlie et al. $(2019)^{40}-$ IIM arm & $\mathrm{RCT}$ & IDA digestive diseases & $0.8 \mathrm{mmol} / \mathrm{L}$ \\
\hline Ding et al. $(2020)^{41}-1000 \mathrm{mg}$ cohort & $\mathrm{RCT}$ & mixed IDA & $0.8 \mathrm{mmol} / \mathrm{L}$ \\
\hline Ding et al. $(2020)^{41}-500 \mathrm{mg}$ cohort & $\mathrm{RCT}$ & mixed IDA & $0.8 \mathrm{mmol} / \mathrm{L}$ \\
\hline Drexler et al. $(2019)^{42}$ & $\mathrm{RCT}$ & IDA/blood donation & $0.84 \mathrm{mmol}(\mathrm{L}$ \\
\hline Evstatiev et al. $(2011)^{43}$ & $\mathrm{RCT}$ & IDA digestive diseases & na \\
\hline Favrat et al. $(2014)^{44}$ & $\mathrm{RCT}$ & mixed IDA & $0.8 \mathrm{mmol} / \mathrm{L}$ \\
\hline Gybel-Brask et al. $(2018)^{45}$ & $\mathrm{RCT}$ & IDA/blood donation & $2 \mathrm{mg} / \mathrm{dL}(0.65 \mathrm{mmol} / \mathrm{L})$ \\
\hline Holm et al. $(2017)^{46}$ & $\mathrm{RCT}$ & GYN & $2 \mathrm{mg} / \mathrm{dL}(0.65 \mathrm{mmol} / \mathrm{L})$ \\
\hline Huang et al. $(2018)^{47}$ - CKD arm & SAT & NDD-CKD & na \\
\hline Huang et al. $(2018)^{47}-$ control arm & SAT & mixed IDA & na \\
\hline Huang et al. $(2018)^{47}$ - pregnant arm & SAT & GYN & na \\
\hline Hussain et al. $(2013)^{48}$ & $\mathrm{RCT}$ & mixed IDA & $2 \mathrm{mg} / \mathrm{dL}(0.65 \mathrm{mmol} / \mathrm{L})$ \\
\hline Ikuta et al. $(2018)^{49}$ & SAT & mixed IDA & $2.5 \mathrm{mg} / \mathrm{dL}(0.81 \mathrm{mmol} / \mathrm{L})$ \\
\hline Ikuta et al. $(2019)^{50}$ & $\mathrm{RCT}$ & GYN & $0.81 \mathrm{mmol} / \mathrm{L}$ \\
\hline Ikuta et al. $(2019)^{51}$ & SAT & IDA digestive diseases & $2.5 \mathrm{mg} / \mathrm{dL}(0.81 \mathrm{mmol} / \mathrm{L})$ \\
\hline Johannsson et al. $(2015)^{52}$ & $\mathrm{RCT}$ & $\mathrm{PBM}$ & $2 \mathrm{mg} / \mathrm{dL}(0.65 \mathrm{mmol} / \mathrm{L})$ \\
\hline Kalra et al. $(2016)^{53}$ - bolus arm & $\mathrm{RCT}$ & NDD-CKD & $2 \mathrm{mg} / \mathrm{dL}(0.65 \mathrm{mmol} / \mathrm{L})$ \\
\hline Kalra et al. $(2016)^{53}$ - infusion arm & $\mathrm{RCT}$ & NDD-CKD & $2 \mathrm{mg} / \mathrm{dL}(0.65 \mathrm{mmol} / \mathrm{L})$ \\
\hline Macdougall et al. $(2014)^{54}-$ high ferritin arm & $\mathrm{RCT}$ & NDD-CKD & na \\
\hline Macdougall et al. $(2014)^{54}$ - low ferritin arm & $\mathrm{RCT}$ & NDD-CKD & na \\
\hline Mahey et al. $(2016)^{55}$ & $\mathrm{RCT}$ & GYN & na \\
\hline Onken et al. $(2014)^{1}-$ cohort 1 & $\mathrm{RCT}$ & mixed IDA & na \\
\hline Onken et al. $(2014)^{1}-$ cohort 2 & $\mathrm{RCT}$ & mixed IDA & na \\
\hline Onken et al. $(2014)^{56}$ & $\mathrm{RCT}$ & NDD-CKD & na \\
\hline Prats et al. $(2013)^{13}$ & $\mathrm{RCT}$ & NDD-CKD & na \\
\hline Qunibi et al. $(2011)^{57}$ & $\mathrm{RCT}$ & NDD-CKD & na \\
\hline Reinisch et al. $(2013)^{9}$ & $\mathrm{RCT}$ & IDA digestive diseases & $2 \mathrm{mg} / \mathrm{dL}(0.65 \mathrm{mmol} / \mathrm{L})$ \\
\hline Roberts et al. $(2016)^{14}$ & $\mathrm{RCT}$ & HD-CKD & na \\
\hline Seid et al. $(2008)^{58}$ & $\mathrm{RCT}$ & GYN & na \\
\hline Seid et al. $(2017)^{59}$ - heavy menstrual bleeding arm & $\mathrm{RCT}$ & GYN & na \\
\hline Seid et al. $(2017)^{59}$ - post-partum anemia arm & $\mathrm{RCT}$ & GYN & na \\
\hline Stohr et al. $(2019)^{60}-$ CKD arm & SAT & NDD-CKD & $0.8 \mathrm{mmol} / \mathrm{L}$ \\
\hline Stohr et al. $(2019)^{60}$ - heart failure arm & SAT & heart failure & $0.8 \mathrm{mmol} / \mathrm{L}$ \\
\hline Toledano et al. $(2016)^{61}$ & SAT & malignancy & na \\
\hline Van Wyck et al. $(2007)^{8}$ & $\mathrm{RCT}$ & GYN & na \\
\hline VanWyck et al. $(2009)^{62}$ & $\mathrm{RCT}$ & GYN & $2 \mathrm{mg} / \mathrm{dL}(0.65 \mathrm{mmol} / \mathrm{L})$ \\
\hline Wolf et al. $(2013)^{15}$ & $\mathrm{RCT}$ & GYN & $2 \mathrm{mg} / \mathrm{dL}(0.65 \mathrm{mmol} / \mathrm{L})$ \\
\hline Wolf et al. $(2018)^{26}$ & $\mathrm{RCT}$ & mixed IDA & $2 \mathrm{mg} / \mathrm{dL}(0.65 \mathrm{mmol} / \mathrm{L})$ \\
\hline Wolf et al. $(2020)^{63}$ - trial A FCM & $\mathrm{RCT}$ & mixed IDA & $2 \mathrm{mg} / \mathrm{dL}(0.65 \mathrm{mmol} / \mathrm{L})$ \\
\hline Wolf et al. $(2020)^{63}$ - trial A IIM & $\mathrm{RCT}$ & mixed IDA & $2 \mathrm{mg} / \mathrm{dL}(0.65 \mathrm{mmol} / \mathrm{L}$ \\
\hline Wolf et al. $(2020)^{63}$ - trial B FCM & $\mathrm{RCT}$ & mixed IDA & $2 \mathrm{mg} / \mathrm{dL}(0.65 \mathrm{mmol} / \mathrm{L})$ \\
\hline Wolf et al. $(2020)^{63}$ - trial B IIM & $\mathrm{RCT}$ & mixed IDA & $2 \mathrm{mg} / \mathrm{dL}(0.65 \mathrm{mmol} / \mathrm{L}$ \\
\hline
\end{tabular}

\section{Figure legends}

Figure1: PRISMA diagram. The search strategy and study selection for the meta-analysis of clinical trials. 
Figure 2: Forest plot on hypophosphatemia rate according to iron preparation and presence of chronic kidney disease.

1 - infusion arm; 2 - bolus arm; 3 - NDD-CKD arm; 4 - bolus arm; 5 - infusion arm; 6 - IIM arm; 7 trial A; 8 - trial B; 9 - single dose arm; 10 - multiple dose arm; 11 - cohort $1 ; 12$ - cohort 2; 13 - heavy menstrual bleeding arm; 14 - post-partum anemia arm; 15 - non-CKD/heart-failure arm; 16 - FCM arm; 17 - Ref: ${ }^{50} ; 18$ - Ref. ${ }^{51} ; 19$ - $500 \mathrm{mg}$ dose; 20 - $1000 \mathrm{mg}$ dose; 21 - trial A; 22 - trial B

Figure 3: Forest plot on severity of hypophosphatemia rate according to iron preparation and presence of chronic kidney disease. (A) Mean lowest serum phosphate concentration. 1 - NDD-CKD arm; 2 - NDD-CKD arm ; 3 - trial B; 4 - trial A; 5 - Ref: ${ }^{51} ; 6$ - Ref: $^{50} ; 7$ - control arm; 8 - pregnant arm; 9 - trial A; 10 - trial B(B) Mean changes in serum phosphate from baseline. 1 - NDD-CKD arm; 2 NDD-CKD arm; 3 - trial B; 4 - trial A; 5 - pregnant arm; 6 - control arm; 7 - non-CKD/heart-failure arm; 8 - trial A; 9 - trial B

Figure 4: Meta regression of hypophosphatemia rate in relation to either $\log$ (mean ferritin) $(\mathrm{A}, \mathrm{B})$ or mean transferrin saturation $(\mathrm{C}, \mathrm{D})$ in studies on FCM $(\mathrm{A}, \mathrm{C})$ or IIM $(\mathrm{B}, \mathrm{D})$. Numerical outputs are reported in Table S1.

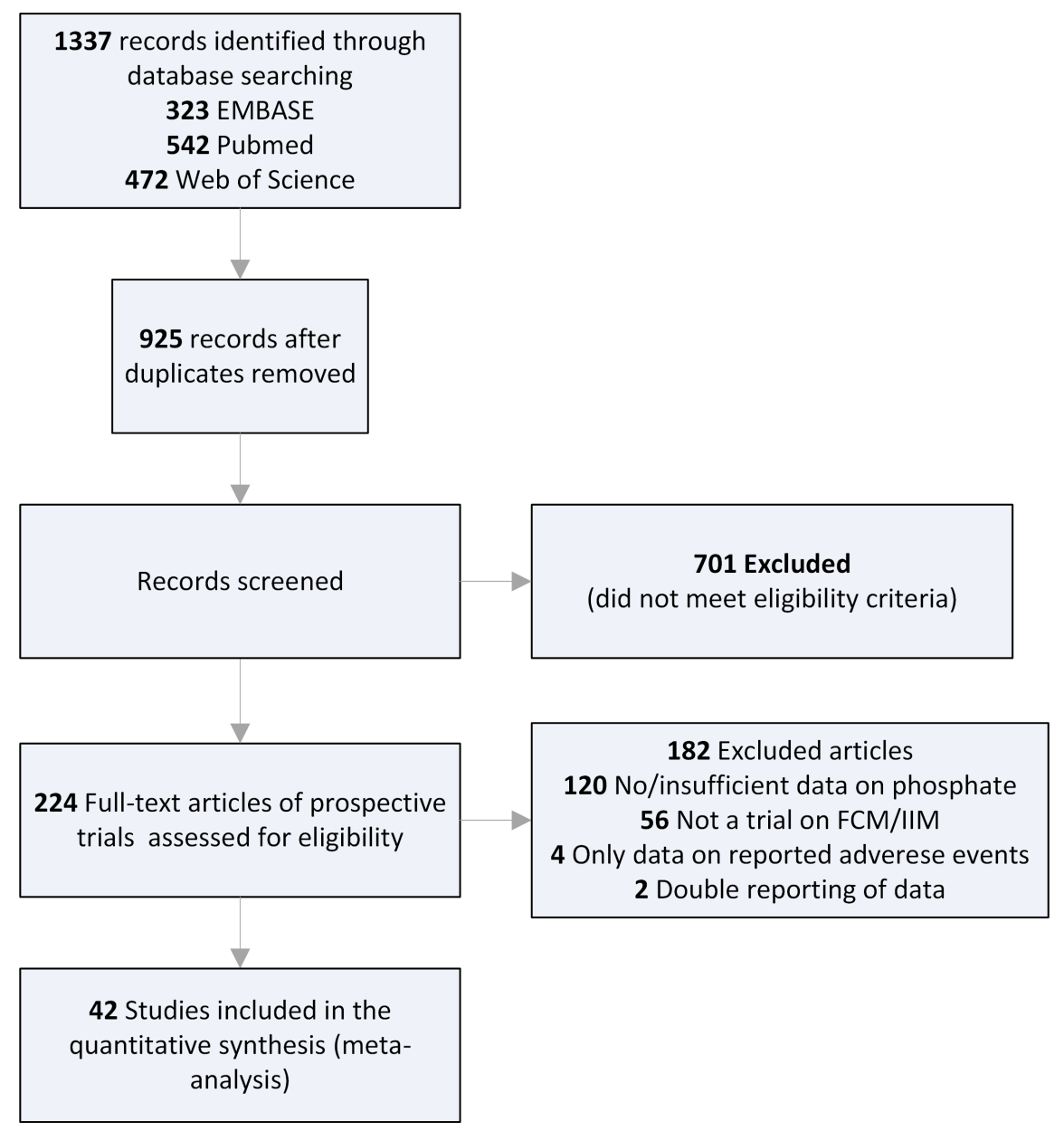




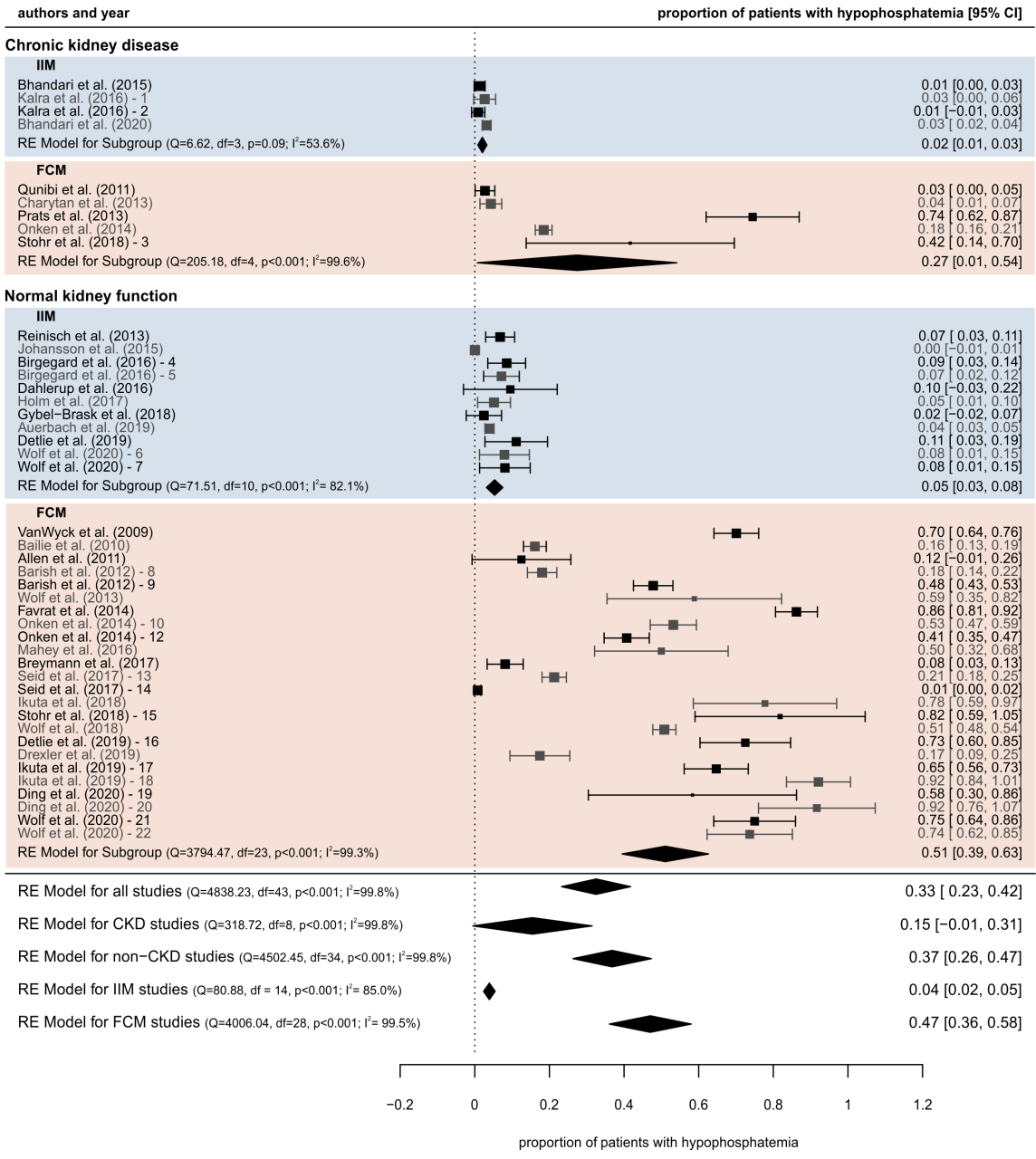


A

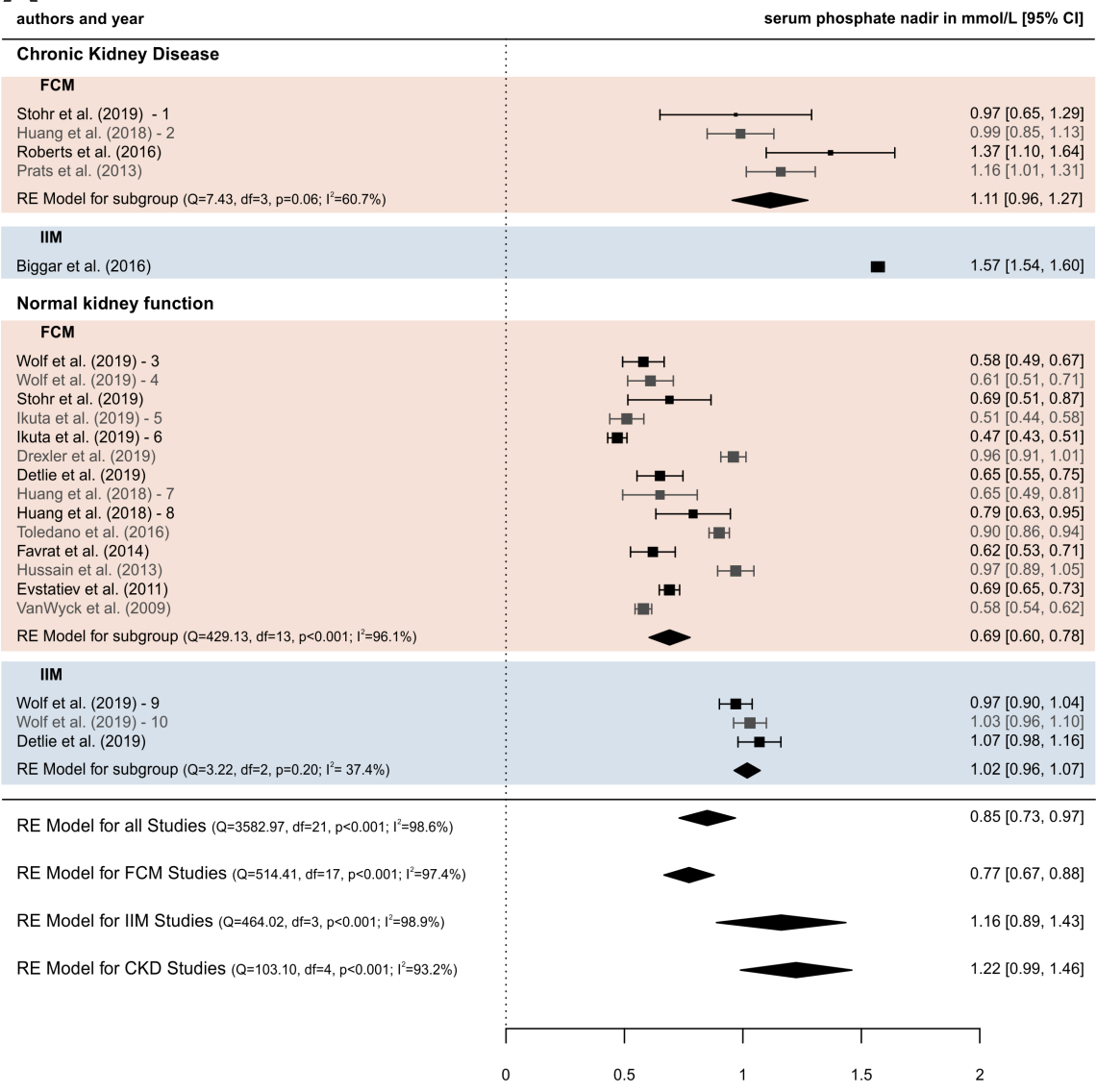

B

Mean lowest serum phosphate [mmo//L]

authors and year

mean change in serum phosphate in $\mathrm{mmol} / \mathrm{L}[95 \% \mathrm{Cl}]$

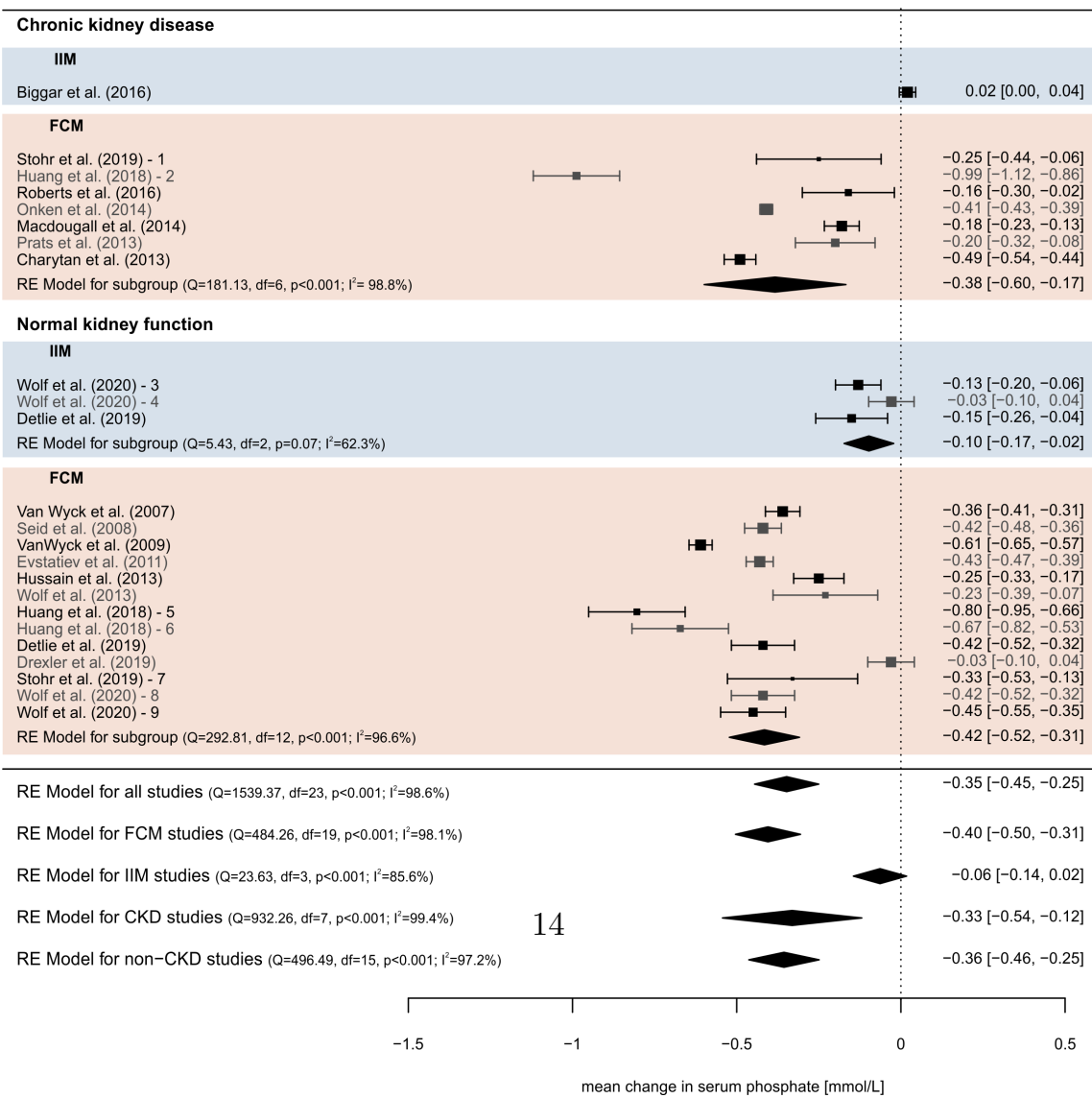



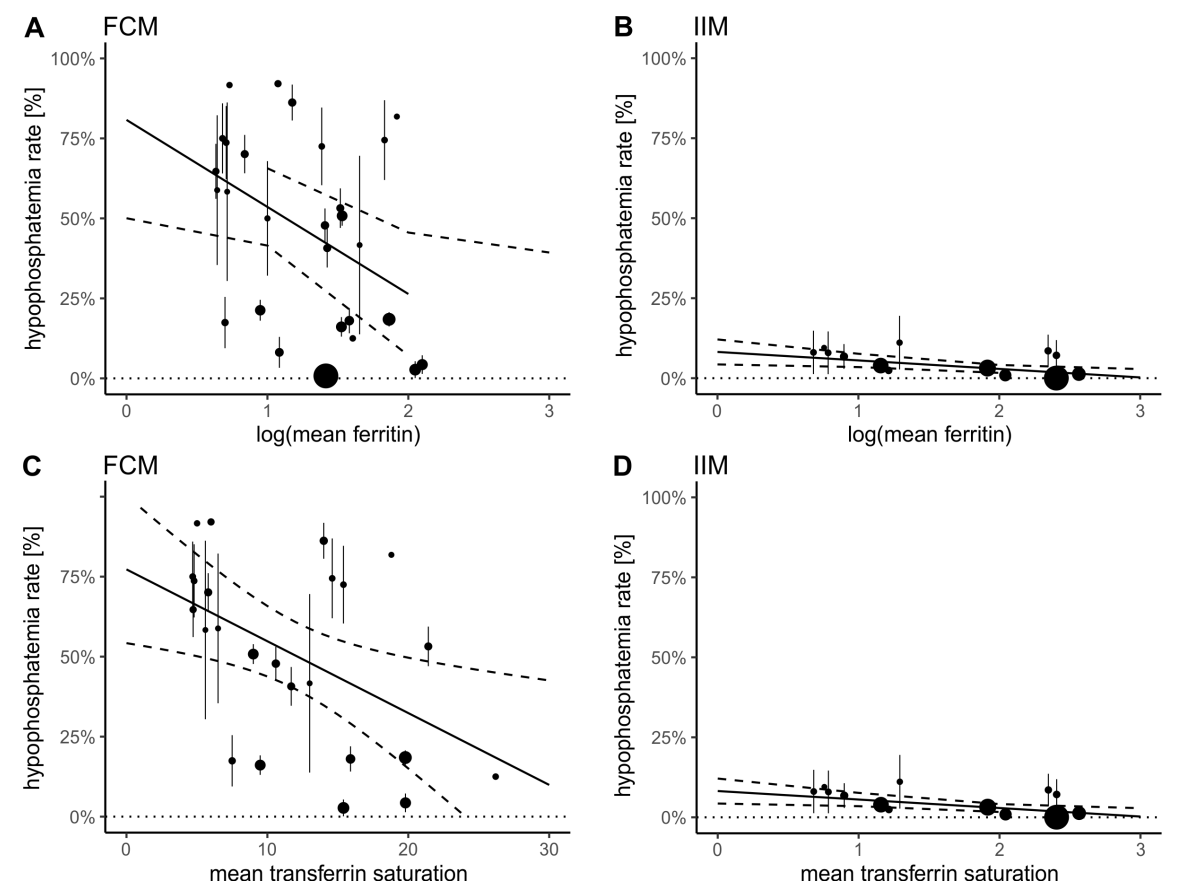